\title{
Combined Hamartoma of the Optic Disc and Retinal Pigment Epithelium-3 Years of the Follow-Up with Semi-Automated Kinetic Perimetry
}

\author{
Katarzyna Nowomiejska ${ }^{1}$, Tomasz Zarnowski ${ }^{2}$, Robert Rejdak ${ }^{1,3,4}$, Anselm Juenemann ${ }^{5}$ \\ ${ }^{1}$ Department of General Ophthalmology, Medical University, Lublin, Poland; ${ }^{2}$ Department of Glaucoma Diagnostics and Microsur- \\ gery, Medical University, Lublin, Poland; ${ }^{3}$ Department of Pathophysiology of Vision and Neuro-Ophthalmology, University Eye \\ Hospital, Tuebingen, Germany; ${ }^{4}$ Department of Experimental Pharmacology, Mossakowski Medical Research Centre, Polish Academy \\ of Sciences, Warsaw, Poland; ${ }^{5}$ Department of Ophthalmology, University of Erlangen-Nürnberg, Erlangen, Germany. \\ Email: katarzynanowomiejska@mailcity.com
}

Received June $21^{\text {st }}$, 2012; revised September $18^{\text {th }}$, 2012; accepted October $7^{\text {th }}, 2012$

\begin{abstract}
Aim: To present a case of hamartoma of the optic disc and Retinal Pigment Epithelium (RPE) and follow up of the visual function over three-years period. Methods: A seventeen-year-old boy has observed reduced visual acuity in his left eye. The visual acuity was 0.2 and there was RAPD in the left eye. Fundoscopy revealed an elevation of the optic disc obscuring disc vessels with epiretinal gliosis. Fluorescein angiography demonstrated hyperfluorescent saccular dilatations with leakage in the late phase. Ocular Coherence Tomography (OCT) showed hyperreflective elevation of the optic disc and epiretinal membrane. There was a juxtapapillary scotoma in Semi-automated Kinetic Perimetry (SKP). There was no systemic diseases. Results of blood tests, CT and MRI of CNV were normal. Results: After 3 years period of the follow-up the visual acuity was 0.1 and there was a progression of the visual field defect to the altitudinal scotoma in the upper hemisphere. Fluorescein anhiography and OCT revealed the same. Conclusion: Hamartoma of RPE and optic disc is a rare condition consisting of glial, vascular and RPE cells. It should be differentiated from optic disc oedema and vascular tumors of the retina. Assessment of the visual function is very important in the longitudinal follow-up.
\end{abstract}

Keywords: Hamartoma; Perimetry; Optic Disc

\section{Case}

A 17-year-old male has observed slow, progressive and painless reduction of the visual acuity of his left eye for two years. He had no systemic diseases, only meningitis after virus infection in childhood (8 years old) was reported. He had no significant family history. The best corrected visual acuity at presentation was 20/20 in the right eye and 20/80 in the left eye. Intraocular pressure was $20 \mathrm{~mm} \cdot \mathrm{Hg}$ in both eyes. Color vision and eye movements were normal. There was RAPD in the left eye. Anterior segment was normal. Fundus was normal in the right eye. In the left eye fundoscopy revealed elevated optic disc margins with scattered hiperpigmentstion obscuring major disc vessels (Figure 1). Additionally, there was an epiretinal gliosis and traction involving the macula. Flurescein angiography (HRA2, Heidelberg Enginering, Germany) demonstrated early hyperfluorescence throughout the lesion and saccular dilatations with leakage in the late phase (Figure 2), OCT (Cirrus HD-OCT Model 400, Carl Zeiss, Meditec, Dublin, CA, USA) revealed the dis- tortion of the retinal architecture, hyperreflective retinal elevation and epiretinal membrane.

Visual field of the right eye was normal, in the left eye juxtapapillary scotoma was observed (Figure 3 left). Perimetry was performed using Semi-automated Kinetic Perimetry (SKP) implemented in Octopus 101 and Octopus 900 instrument. MRI and CT examinations were described as normal. In the neurological examination no pathological signs were observed. The systemic studies produced no abnormalities.

Three years later the visual acuity was 0.1 in the left eye, the visual field has progressed to altitudinal scotoma in the upper hemisphere (Figure 3 right). The fundus and fluorescein angiography appeared the same. There was no treatment applied.

\section{Discussion}

Hamartoma of the optic disc and Retinal Pigment Epithelium (RPE) is a rare benign congenital ocular tumor described first by Gass [1]. It is single, solitary and unila- 


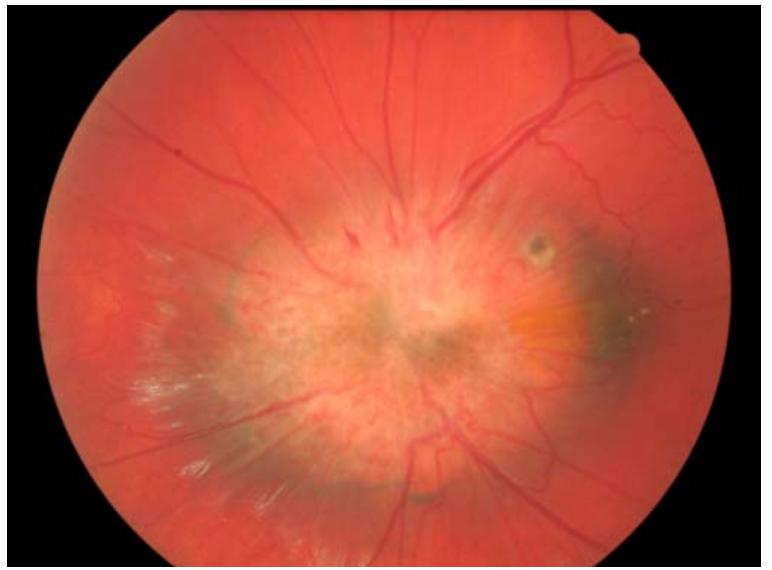

Figure 1. Fundus photograph of the left eye showing pigment epithelium hamartoma of the optic disc.
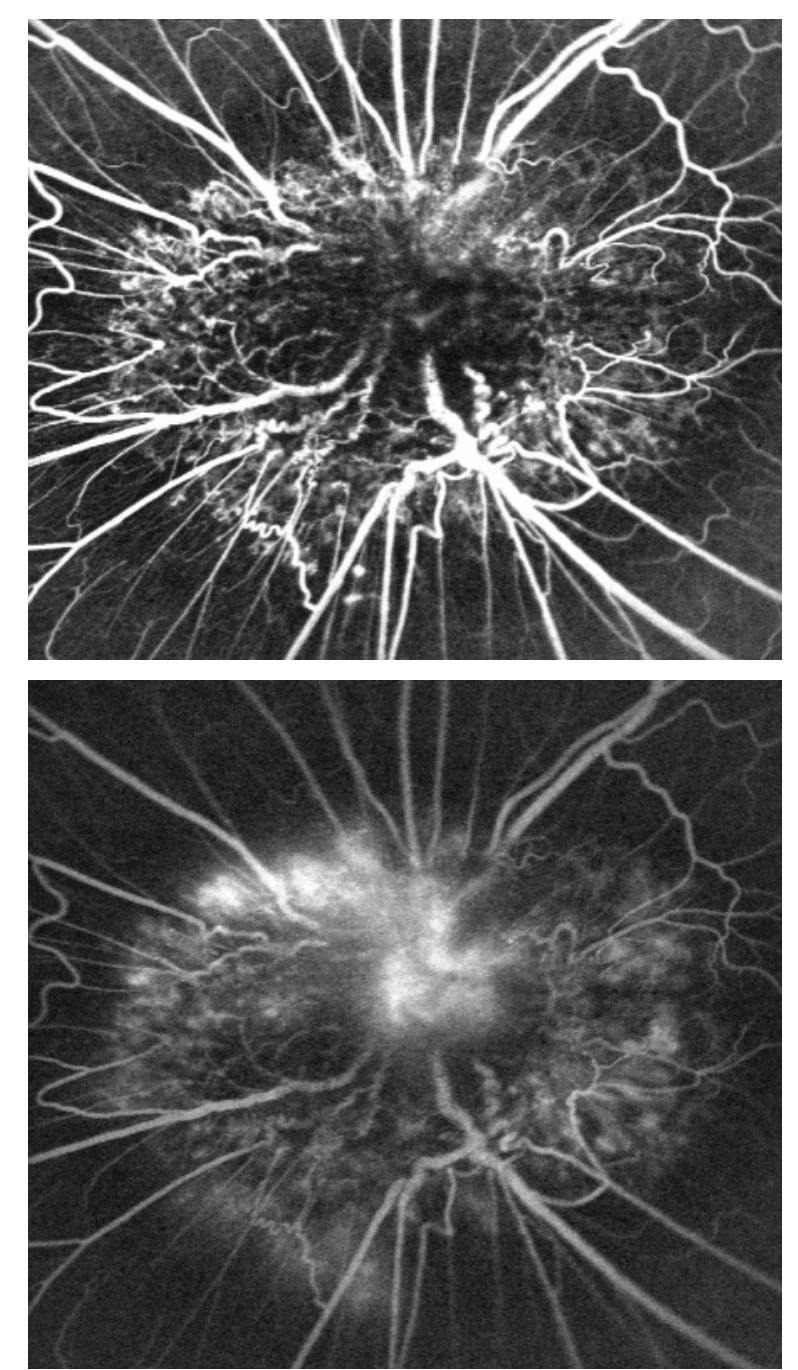

Figure 2. Fluorescein angiography (Spectralis HRA2, Heidelberg Enginering, Germany) of the left eye showing early phase (0:21 min.) - at the top and late phase with leakage (4:51 min.) - below.
LA
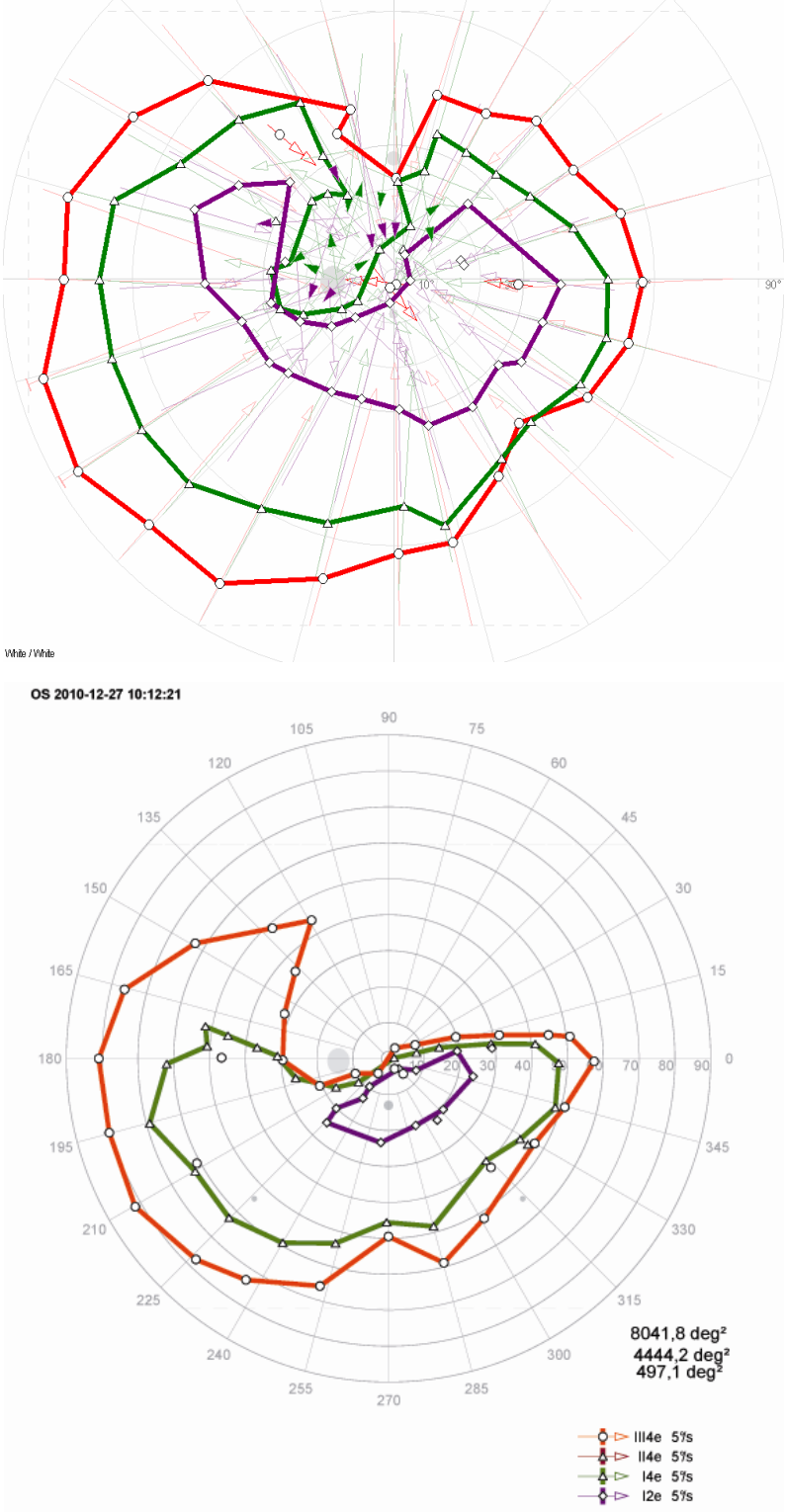

Figure 3. Result of semi-automated kinetic perimetry of the left eye. Three isopters I2e, I4e and III4e. Baseline examination (Octopus 101) - juxtapapillary scotoma (top). Perimetry after three years (Octopus 900)—altitudinal scotoma in the upper hemisphere (bottom).

teral intraocular tumor. Histologically, it is composed of three cell populations: pigmented, glial and vascular. Different proportions of these cells are responsible for various clinical pictures. Usually hamartoma of the retina and RPE are located close to the optic disc in $76 \%$, in the macula in $17 \%$ and in the peripheral retina in $17 \%$. Only few cases have been described so far localized within the optic nerve head [2]. 
The diagnostics is based on fundoscopy and confirmed by fluorescein angiography and Ocular Coherence Tomography (OCT). The presenting syndrome is painless unilateral silent visual loss. The patients are usually asymptomatic until the late childhood. The anterior segment is not affected. There are anecdotical reports on systemic associations with neurofibromatosis type II and tuberous sclerosis. RPE hamartomas usually show no growth potential. Hamartoma of the retina and RPE can generate a large papillary and retinal distortion [3], thus it is often accompanied with vascular tortuosity and epiretinal membrane. Secondary changes as vitreous hemorrhage, Choroidal Neovascular Membrane (CNV) or retinal detachment [4] on some occasions may cause visual loss. There have been reports describing pars plana vitrectomy due to epiretinal membrane following hamartoma [5] or submacular surgery due to CNV [6].

In our case there was a slight progression of the visual function - both visual acuity and visual field during three years of the follow-up. The visual field defect has extended to the periphery. It was well documented using SKP. This method enables examination of the entire visual field using moving stimuli. The results are comparable to Goldmann manual kinetic perimetry [7], however it is more standardized and not so examiner-dependent.

The structural examinations as OCT and fluorescein angiography appeared the same.

In conclusion, hamartoma of RPE and optic disc could be a vision devastating tumor. Differential diagnosis should include papilloedema, vascular tumors (hemangioma), primary optic disc tumors as astrocytoma and melanocytoma, secondary optic disc tumors as metastatic tumor and leukemia and tumors invading the optic nerve from adjacent structures (retinoblastoma, choroidal melanoma and meningioma) [8]. It is very important to differentiate these conditions as there are specific systemic associations, treatment and prognosis associated with them. Hamartoma may be also confused with teratoma, composed of tissue foreign to the site of growth. The major difference is that teratoma can be increasing while hamartoma usually is not sustained growth.

Regular follow-up of the structure and function is necessary for successful management of hamartoma of the optic nerve and RPE. To monitor carefully the visual function, especially the peripheral visual field, it seems to be advisable to perform SKP in patients with this rare condition.

\section{REFERENCES}

[1] J. D. Gass, “An Unusual Hamartoma of the Pigment Epithelium and Retina Simulating Choroidal Melanoma and Retinoblastoma," Transactions of the American Ophthalmological Society, Vol. 71, 1973, pp. 175-183.

[2] R. L. Font, R. A. Moura, D. J. Shetlar, et al., "Combined Hamartoma of Sensory Retina and Retinal Pigment Epithetlium,” Retina, Vol. 9, No. 4, 1989, pp. 302-311. doi:10.1097/00006982-198909040-00011

[3] M. J. Pérez-Alvarez, N. Alejandre-Alba and J. Gar- cíaSánchez, "Combined Hamartoma of the Retina and Retinal Pigment Epithelium Diagnosed by Retinal Angiography and Optical Coherence Tomography," Archivos de la Sociedad Española de Oftalmología Vol. 83, No. 3, 2008, pp. 193-196. doi:10.4321/S0365-66912008000300011

[4] H. Helbig and H. Niederberger, "Presumed Combined Hamartoma of the Retina and Retinal Pigment Epithelium with Preretinal Neovascularization," American Journal of Ophthalmology, Vol. 136, No. 6, 2003, pp. 1157-1159. doi:10.1016/S0002-9394(03)00568-3

[5] X. Zhang, F. Dong, R. Dai, et al., "Surgical Management of Epiretinal Membrane in Combined Hamartomas of the Retina and Retinal Pigment Epithelium,” Retina, Vol. 30, No. 2, 2010, pp. 305-309. doi:10.1097/IAE.0b013e3181b85f2d

[6] M. Inoue, K. Noda, S. Ishida, et al., "Successful Treatment of Subfoveal Choroidal Neovascularization Associated with Combined Hamartoma of the Retina and Retinal Pigment Epithelium," American Journal of Ophthalmology, Vol. 138, No. 1, 2004, pp. 155-156.

[7] K. Nowomiejska, R. Vonthein, J. Paetzold, et al., "Comparison Between Semiautomated Kinetic Perimetry and Conventional Goldmann Manual Kinetic Perimetry in Advanced Visual Field Loss,” Ophthalmology, Vol. 112, No. 8, 2005, pp. 343-354. doi:10.1016/j.ophtha.2004.12.047

[8] G. C. Brown and J. A. Shields, "Tumors of the Optic Nerve Head," Survey of Ophthalmology, Vol. 29, No. 4, 1985, pp. 239-264. doi:10.1016/0039-6257(85)90149-3 